\title{
Drift wave excitation in a collisional dusty magnetoplasma with multi-ion species
}

\author{
P. K. SH UK L A ${ }^{1} \dagger$ and M. R O SE N B R G \\ ${ }^{1}$ Department of Electrical and Computer Engineering, University of California, \\ San Diego, La Jolla, CA 92093, USA
}

(Received 13 October 2008 and accepted 3 November 2008, first published online 15 December 2008)

\begin{abstract}
We investigate the drift dissipative instability in a non-uniform magnetized plasma composed of electrons, positive ions, negative ions and negatively charged dust particles. We use a multi-fluid plasma model and derive a dispersion relation for the electrostatic drift waves with frequencies much smaller than the ion gyrofrequencies and wavelengths longer than the ion gyroradii. The presence of the negatively charged, massive dust grains affects the drift wave frequency and the growth rate of the drift dissipative instability. The present results may be relevant to space and laboratory magnetoplasmas containing negative ions and charged dust grains.
\end{abstract}

The low-frequency electrostatic drift wave is one of the fundamental modes of a non-uniform magnetized plasma (e.g. [1-4]). In the standard theory of the drift dissipative instability, consideration of a non-Boltzmann electron density perturbation owing to electron-ion collisions leads to a phase shift between the electron density perturbation and the wave potential, which in turn produces instability $[1-6]$. Non-thermal drift waves cause cross-field transport [5] of the electrons and ions in an inhomogeneous magnetoplasma. Furthermore, it is well known [7-10] that dispersive drift waves, driven by the drift dissipative instability, can be responsible for the formation of zonal flows, streamers, and vortical structures. The latter play an important role in tokamaks [11], for example.

It has been shown that the properties of the dispersive drift wave [12] can be modified in the presence of charged dust grains $[13,14]$ owing to the modification of the equilibrium quasi-neutrality condition and the dust particle dynamics. Dust charge fluctuations can also modify the properties of electrostatic drift waves [15, 16]. The dynamics of the collisional drift wave instability in an electron-ion plasma with stationary dust grains (i.e. dust grains that do not move on the time scale of the instability) has been studied in [17], without presenting, the modification of the instability growth rate and the drift wave frequency owing to the presence of dust.

$\dagger$ Permanent address: Institut für Theoretische Physik IV, Fakultät für Physik und Astronomie, Ruhr-Universität Bochum, D-44780 Bochum, Germany

(ps@tp4.ruhr-uni-bochum.de). 
In this letter, we present an investigation of the drift dissipative instability in a non-uniform magnetoplasma whose constituents are the electrons, positive ions, negative ions and negatively charged dust grains that are stationary and non-uniformly distributed. We use a fluid theory description. Namely, we use the continuity equations for the electrons and the two ion species, together with the perpendicular components of the electron and ion fluid velocities in the drift approximation, together with the parallel component of the electron equation of motion and the quasi-neutrality condition to derive the modified ion vorticity equation. The latter is Fourier analyzed to obtain the local dispersion relation, which yields the dispersive drift wave frequency and the growth rate of the dissipative drift wave instability. Both the drift wave frequency and the growth rate are significantly modified by the presence of the negative ions and the dust. The relevance of our investigation to space and laboratory magnetoplasmas is mentioned.

We consider a non-uniform dusty magnetoplasma composed of electrons, positive and negative ions and negatively charged dust grains. The external magnetic field is $B_{0} \hat{z}$, where $B_{0}$ is the magnetic field strength and $\hat{z}$ is a unit vector along the $z$ axis in a Cartesian coordinate system. The equilibrium density $n_{j 0}(x)$ has a gradient $\partial n_{j 0} / \partial x$, where the subscript $j=\mathrm{e},+,-$, d stands for the electrons, positive ions, negative ions and negatively charged dust, respectively. At equilibrium, we have

$$
n_{\mathrm{e} 0}(x)+Z_{\mathrm{d}} n_{\mathrm{d} 0}(x)=Z_{+} n_{+0}(x)-Z_{-} n_{-0}(x),
$$

where $Z_{\mathrm{d}}, Z_{-}$and $Z_{+}$are, respectively, the number of electrons residing on each dust grain, the charge state of the positive ions and the charge state of the negative ions. It is assumed that density gradients are maintained by some external sources, that all species are magnetically confined, and that the density of each species decreases in the $x$-direction.

We consider low frequencies such that $\nu_{\mathrm{i}} \ll \omega \ll \nu_{\mathrm{e}} \ll \omega_{c \mathrm{e}}$, where $\nu_{\mathrm{e}}$ and $\nu_{\mathrm{i}}$ are the electron and ion collision frequencies, respectively, and $\omega_{c \mathrm{e}}=e B_{0} / m_{\mathrm{e}} c$ is the electron gyrofrequency, where $e$ is the magnitude of the electron charge, $m_{\mathrm{e}}$ is the electron mass and $c$ is the speed of light in vacuum. We also consider long wavelengths such that the wavelength $\lambda$ is much greater than the ion gyroradii. For electrostatic waves with $\mathbf{E}=-\nabla \phi$, where $\phi$ is the scalar potential, the electron and ion fluid velocities are, respectively,

$$
\mathbf{v}_{\mathrm{e}} \approx \frac{c}{B_{0}}(\hat{z} \times \nabla \phi)-\frac{c T_{\mathrm{e}}}{e B_{0} n_{\mathrm{e}}}\left(\hat{z} \times \nabla n_{\mathrm{e}}\right)+\hat{z} v_{\mathrm{e} z}
$$

and

$$
\mathbf{v}_{ \pm} \approx \frac{c}{B_{0}}(\hat{z} \times \nabla \phi) \pm \frac{c T_{\mathrm{i}}}{e B_{0} n_{ \pm}}\left(\hat{z} \times \nabla n_{ \pm}\right) \mp \frac{c}{B_{0} \omega_{ \pm}}\left(\frac{\partial}{\partial t}+v_{* \pm} \frac{\partial}{\partial y}\right) \nabla_{\perp} \phi .
$$

Here $T_{\mathrm{e}}\left(T_{\mathrm{i}}\right)$ is the electron (ion) temperature, $n_{\mathrm{e}}\left(n_{ \pm}\right)$is the electron (ion) number density, $\omega_{c \pm}=Z_{ \pm} e B_{0} / m_{ \pm} c$ is the ion gyrofrequency, and $m_{ \pm}$is the ion mass. We have denoted $v_{* \pm}=\left(c T_{\mathrm{i}} / Z_{ \pm} e B_{0} n_{ \pm 0}\right)$. The third term on the right-hand side of (3) is the ion polarization drift. The ion motion parallel to $\hat{z}$ has been neglected, which amounts to discarding dust-modified ion-acoustic waves $[18,19]$ from our plasma system. 
The parallel (to $\hat{z}$ ) component of the electron fluid velocity perturbation $v_{\mathrm{e} z}$ is obtained from the inertialess electron momentum equation

$$
\nu_{\mathrm{e}} v_{\mathrm{e} z} \approx \frac{e}{m_{\mathrm{e}}} \frac{\partial}{\partial z}\left(\phi-\frac{T_{\mathrm{e}}}{e} \frac{n_{\mathrm{e} 1}}{n_{\mathrm{e} 0}}\right),
$$

where $n_{\mathrm{e} 1}\left(=n_{\mathrm{e}}-n_{\mathrm{e} 0}(x)\right)$ is a small perturbation in the equilibrium electron number density $n_{\mathrm{e} 0}(x)$. The electron density perturbation is obtained from the linearized electron continuity equation and (2). We have

$$
\frac{\partial n_{\mathrm{e} 1}}{\partial t}=\frac{e}{B_{0}} \frac{d n_{\mathrm{e} 0}}{d x} \frac{\partial \phi}{\partial y}+n_{\mathrm{e} 0} \frac{\partial v_{\mathrm{e} z}}{\partial z}=0
$$

Letting $n_{ \pm}=n_{ \pm 0}(x)+\tilde{n}_{ \pm}$, where $\tilde{n}_{ \pm} \ll n_{ \pm 0}$, in the ion continuity equation, and using (3) we have

$$
\frac{\partial \tilde{n}_{ \pm}}{\partial t}-\frac{c}{B_{0}} \frac{d n_{ \pm 0}}{d x} \frac{\partial \phi}{\partial y} \mp \frac{n_{ \pm 0} c}{B_{0} \omega_{c \pm}}\left(\frac{\partial}{\partial t}+v_{* \pm} \frac{\partial}{\partial y}\right) \nabla_{\perp}^{2} \phi=0 .
$$

Multiplying the positive (negative) ion continuity equation above by $Z_{+}\left(Z_{-}\right)$, subtracting the resulting negative ion equation from the positive ion equation, and using the quasi-neutrality condition

$$
n_{\mathrm{e} 1}=Z_{+} \tilde{n}_{+}-Z_{-} \tilde{n}_{-},
$$

we obtain

$$
\frac{\partial n_{\mathrm{e} 1}}{\partial t}-\frac{c}{B_{0}} \frac{d N_{0}}{d x} \frac{\partial \phi}{\partial y}-\frac{c}{B_{0}} \sum_{+,-} \frac{Z_{ \pm} n_{ \pm 0}}{\omega_{c \pm}} \frac{\partial}{\partial t} \nabla_{\perp}^{2} \phi=0,
$$

where we have assumed that $\rho_{ \pm}^{2}\left|\nabla_{\perp}^{2} \phi\right| \ll \phi$. Here $\rho_{ \pm}=v_{\mathrm{T} \pm} / \omega_{c \pm}$ is the ion gyroradius and $v_{\mathrm{T} \pm}$ is the ion thermal speed. We have denoted $N_{0}(x)=n_{\mathrm{e} 0}(x)+Z_{\mathrm{d}} n_{\mathrm{d} 0}(x)$.

Equations (4), (5) and (8) are the desired equations for the study of the driftdissipative instability in a collisional dusty magnetoplasma with multi-ion species. From (4) and (5) we have

$$
\left(\frac{\partial}{\partial t}-D_{\mathrm{e}} \frac{\partial^{2}}{\partial z^{2}}\right) n_{\mathrm{e} 1}-\frac{c}{B_{0}} \frac{d n_{\mathrm{e} 0}}{d x} \frac{\partial \phi}{\partial y}+\frac{n_{\mathrm{e} 0} e}{m_{\mathrm{e}} \nu_{\mathrm{e}}} \frac{\partial^{2} \phi}{\partial z^{2}}=0
$$

where $D_{\mathrm{e}}=v_{\mathrm{Te}}^{2} / \nu_{\mathrm{e}}$ represents the collisional electron diffusion coefficient and $v_{\mathrm{Te}}=$ $\left(T_{\mathrm{e}} / m_{\mathrm{e}}\right)^{1 / 2}$ is the electron thermal speed.

Now, by using (9) we can eliminate $n_{\mathrm{e} 1}$ from (8), obtaining the wave equation

$$
\begin{aligned}
& \frac{c}{B_{0}} \frac{d}{d x}\left(Z_{\mathrm{d}} n_{\mathrm{d} 0}\right) \frac{\partial^{2} \phi}{\partial y \partial t}-\frac{c}{B_{0}} D_{\mathrm{e}} \frac{d N_{0}}{d x} \frac{\partial^{3} \phi}{\partial z^{2} \partial y} \\
& \quad+\frac{c}{B_{0}}\left(\sum_{+,-} \frac{Z_{ \pm} n_{ \pm 0}}{\omega_{c \pm}}\right)\left(\frac{\partial}{\partial t}-D_{\mathrm{e}} \frac{\partial^{2}}{\partial z^{2}}\right) \nabla_{\perp}^{2} \frac{\partial \phi}{\partial t}+\frac{n_{\mathrm{e} 0} e}{m_{\mathrm{e}} \nu_{\mathrm{e}}} \frac{\partial^{3} \phi}{\partial z^{2} \partial t}=0 .
\end{aligned}
$$

Ignoring the magnetic field aligned motion, we obtain from (10)

$$
\frac{\partial}{\partial t} \nabla_{\perp}^{2} \phi+\left(\sum_{+,-} \frac{Z_{ \pm} n_{ \pm 0}}{\omega_{c \pm}}\right)^{-1} \frac{d}{d x}\left(Z_{\mathrm{d}} n_{\mathrm{d} 0}\right) \frac{\partial \phi}{\partial y}=0
$$

which governs the Shukla-Varma mode [20] in a non-uniform dusty magnetoplasma with two ion species. 
Supposing that $\phi=\phi \exp \left(-i \omega t+i \mathbf{k}_{\perp} \cdot \mathbf{r}_{\perp}+i k_{z} z\right)$, where $\omega$ and $\mathbf{k}\left(=\mathbf{k}_{\perp}+\hat{z} k_{z}\right)$ are the frequency and wavevector, respectively, we Fourier analyze (10) to obtain the local dispersion relation:

$$
\left(1+k_{\perp}^{2} \rho_{s}^{2}\right) \omega+k_{y} V_{*}-\frac{i \omega \nu_{\mathrm{e}}}{n_{\mathrm{e} 0} \omega_{c \mathrm{e}}} \frac{k_{y}}{k_{z}^{2}} \frac{d\left(Z_{\mathrm{d}} n_{\mathrm{d} 0}\right)}{d x}-\frac{i \omega^{2} \nu_{\mathrm{e}}}{\omega_{c \mathrm{e}}} \frac{k_{\perp}^{2}}{k_{z}^{2}}\left(\sum_{+,-} \frac{Z_{ \pm} n_{ \pm 0}}{n_{\mathrm{e} 0} \omega_{c \pm}}\right)=0,
$$

where $V_{*}=\left(D_{B} / n_{\mathrm{e} 0}\right)\left(d N_{0} / d x\right), D_{B}=c T_{\mathrm{e}} / e B_{0}$, and

$$
\rho_{s}=\left(D_{B} \sum_{+,-} \frac{Z_{ \pm} n_{ \pm 0}}{n_{\mathrm{e} 0} \omega_{c \pm}}\right)^{1 / 2}
$$

is the effective ion sound gyroradius in our dusty plasma with multi-ion species. We note that (12) is valid as long as the gradient scale sizes are much longer than the wavelength.

Letting $\omega=\omega_{k}+i \gamma_{k}$, where $\gamma_{k} \ll \omega_{k}$, we obtain the drift wave frequency

$$
\omega_{k}=-\frac{D_{B} k_{y}\left(d N_{0} / d x\right)}{n_{\mathrm{e} 0}\left(1+k_{\perp}^{2} \rho_{s}^{2}\right)},
$$

and the growth rate

$$
\gamma_{k}=\frac{\nu_{\mathrm{e}}}{\omega_{c \mathrm{e}}} \frac{\omega_{k}}{\left(1+k_{\perp}^{2} \rho_{s}^{2}\right)}\left[\omega_{k} \frac{k_{\perp}^{2}}{k_{z}^{2}}\left(\sum_{+,-} \frac{Z_{ \pm} n_{ \pm 0}}{n_{\mathrm{e} 0} \omega_{c \pm}}\right)+\frac{k_{y}}{k_{z}^{2}} \frac{d\left(Z_{\mathrm{d}} n_{\mathrm{d} 0}\right) / d x}{n_{\mathrm{e} 0}}\right] .
$$

We note that in the limit where the dust charge density is much smaller than that of the ions, the presence of a high concentration of negative ions, with $Z_{-} n_{-0} / n_{\mathrm{e} 0}>1$, would lead to a decrease in the drift wave frequency because the effective ion sound gyroradius increases. In this case, the growth rate would tend to increase because it is proportional to $Z_{-} n_{-0} / n_{\mathrm{e} 0}$. On the other hand, when the dust charge density is much larger than the negative ion charge density and when $Z_{\mathrm{d}} n_{\mathrm{d} 0} / n_{\mathrm{e} 0}>1$, the frequency can increase, while the growth rate can decrease. (Note that since we are considering a plasma in which all species are magnetically confined, and the charge density of each species decreases with $x$, that is, $Z_{\mathrm{d}} n_{\mathrm{d} 0} / d x<0$ ). The latter may come about because in the approximation we are considering, where the dust is stationary, the portion of the negative charge density that is residing on the grains does not get perturbed, so there is less of a charge separation in the density perturbation arising from the ion inertia (see also [21]).

In the presence of enhanced drift wave turbulence, there appears a cross-field electron flux

$$
\Gamma_{x}=\left\langle n_{\mathrm{e} 1} v_{\mathrm{e} x}^{*}\right\rangle+\text { complex conjugate, }
$$

where the angular bracket denotes the ensemble average and the asterisk denotes the complex conjugate here. The electron density perturbation is

$$
n_{\mathrm{e} 1}=-\frac{k_{y} c}{\omega B_{0}} \frac{d N_{0}}{d x} \phi_{k} .
$$

The $x$ component of the electron fluid velocity is

$$
v_{\mathrm{e} x} \approx-i \frac{k_{y} c}{B_{0}} \phi_{k}
$$


Hence, the drift wave fluctuation driven cross-field electron flux becomes

$$
\Gamma_{x}=-D_{\mathrm{T}} \frac{d N_{0}}{d x}
$$

where the anomalous diffusion coefficient is

$$
D_{\mathrm{T}}=\frac{2 c^{2}}{B_{0}^{2}} \sum_{\mathbf{k}} \frac{k_{y}^{2} \gamma_{k}\left|\phi_{k}\right|^{2}}{\left(\omega_{k}^{2}+\gamma_{k}^{2}\right)} .
$$

It follows that $D_{\mathrm{T}}$ is determined provided that the saturated fluctuation spectrum $\left|\phi_{k}\right|^{2}$ is either known experimentally or theoretically.

To summarize, we have presented an investigation of drift-dissipative instability in a non-uniform multi-component dusty magnetoplasma composed of the inertialess collisional electrons, magnetized positive and negative ions and stationary charged dust grains that are non-uniformly distributed. By using the multi-fluid equations for the plasma particles, we have derived the wave equation (10). The effects of the charged dust grains appear through the equilibrium quasi-neutrality (1), which allows the possibility of a non-vanishing divergence of the particle flux involving the $\left(c / B_{0}\right) \hat{\mathbf{z}} \times \nabla \phi$ drift owing to the presence of dust. Equation (10) reveals that the collisional, finite $k_{z}$ and dust density gradient effects considerably modify the local dispersion relation (12). The drift wave frequency and the growth rate, which are given by (13a) and (13b), are significantly modified by the presence of negative dust grains and two components of ions. Specifically, there are modifications of $V_{*}$ and $\rho_{s}$. A negative dust density gradient contributes to the growth rate of the drift-dissipative instability. Furthermore, a spectrum of non-thermal drift wave fluctuations is found to produce cross-field electron diffusion in our nonuniform multi-component collisional dusty magnetoplasma. The present results are useful for understanding the salient features of the low-frequency dispersive drift waves and associated cross-field electron and ion transports in magnetized dusty space and laboratory plasmas.

\section{Acknowledgement}

This research was supported by DOE grant no. DE-FG02-04ER54804.

\section{References}

[1] Kadomtsev, B. B. 1965 Plasma Turbulence. New York: Academic Press.

[2] Shukla, P. K., Yu, M. Y., Rahman, H. U. et al. 1981 Phys. Rev. A 23, 321.

[3] Shukla, P. K. and Stenflo, L. 2002 Europhys. J. D $20,103$.

[4] Shukla, P. K., Yu, M. Y., Rahman, H. U. et al. 1984 Phys. Rep. 105, 229.

[5] Weiland, J. 2000 Collective Modes in Inhomogeneous Plasmas. Bristol: Institute of Physics.

[6] Vranjes, J. and Poedt, S. 2008 Phys. Plasmas 15, 034504.

[7] Hasegawa, A. and Wakatani, M. 1983 Phys. Rev. Lett. 50, 682.

[8] Wakatani, M. and Hasegawa, A. 1984 Phys. Fluids 27, 611.

[9] Holland, C. et al. 2006 Phys. Rev. Lett. 96, 195002.

[10] Yan, Z., Yu, J. H., Holland, C., Xu, M., Müller, S. H. and Tynan, G. R. 2008 Phys. Plasmas 15, 092309.

[11] Tsytovich, V. N. and Winter, J. 1998 Phys. Usp. 41, 815.

[12] Shukla, P. K., Yu, M. Y. and Bharuthram, R. 1991 J. Geophys. Res. 96, 21343. 
[13] Shukla, P. K. and Mamun, A. A. 2002 Introduction to Dusty Plasma Physics. Bristol: Institute of Physics.

[14] Shukla, P. K. and Mamun, A. A. 2003 New J. Phys. 5, 17.

[15] Benkadda, S., Tsytovich, V. N. and Verga, A. 1995 Comm. Plasma Phys. Control. Fusion 16, 321 .

[16] Benkadda, S. and Tsytovich, V. N. 2002 Plasma Phys. Rep. 28, 395.

[17] Benkadda, S., Gabbai, P., Tsytovich, V. N. and Verga, A. 1996 Phys. Rev. E 53, 2717.

[18] Shukla, P. K. and Silin, V. P. 1992 Phys. Scr. 45, 508.

[19] Bharuthram, R., Saleem, H. and Shukla, P. K. 1992 Phys. Scr. 45, 512.

[20] Shukla, P. K. and Varma, R. K. 1993 Phys. Fluids B 5, 236.

[21] Siva Rama Prasad, P. V. 1997 Phys. Lett. A 235, 610. 